\title{
Stability and Symmetry Breaking in Metal Nanowires
}

\author{
D. F. Urban ${ }^{1}$, J. Bürki ${ }^{2}$, C. A. Stafford ${ }^{2}$, and Hermann Grabert ${ }^{1}$ \\ ${ }^{1}$ Physikalisches Institut, Albert-Ludwigs-Universität, D-79104 Freiburg, Germany \\ ${ }^{2}$ Department of Physics, University of Arizona, Tucson, AZ 85721
}

(Dated: August 16, 2006)

\begin{abstract}
A general linear stability analysis of simple metal nanowires is presented using a continuum approach which correctly accounts for material-specific surface properties and electronic quantum-size effects. The competition between surface tension and electron-shell effects leads to a complex landscape of stable structures as a function of diameter, cross section, and temperature. By considering arbitrary symmetry-breaking deformations, it is shown that the cylinder is the only generically stable structure. Nevertheless, a plethora of structures with broken axial symmetry is found at low conductance values, including wires with quadrupolar, hexapolar and octupolar cross sections. These non-integrable shapes are compared to previous results on elliptical cross sections, and their material-dependent relative stability is discussed.
\end{abstract}

PACS numbers: 62.25.+g, 47.20.Dr, 68.65.La

\section{INTRODUCTION}

Free-standing metal nanowires, suspended from electrical contacts at their ends, have been fabricated by a number of different techniques. ${ }^{1}$ Metal wires down to a single atom thick were extruded using a scanning-tunneling microscope tip. ${ }^{2,3}$ Metal nanobridges were shown to "selfassemble" under electron-beam irradiation of thin metal films, ${ }^{4,5,6}$ leading to nearly perfect cylinders down to four atoms in diameter, with lengths up to fifteen nanometers. Systematic studies of nanowire properties for a variety of materials were carried out using the mechanicallycontrollable break junction technique. ${ }^{7,8,9,10,11,12}$ As the ultimate nanoscale conductors, metal nanowires are of great interest for nanotechnology.

A remarkable feature of metal nanowires is that they are stable at all. Most atoms in such a thin wire are at the surface, with small coordination numbers, so that surface effects play a key role in their energetics. Indeed, macroscopic arguments comparing the surface-induced stress to the yield strength indicate a minimum radius for solidity of order ten nanometers. ${ }^{13}$ Below this critical radius, plastic flow would lead to a Rayleigh instability, ${ }^{14}$ absent some other stabilizing mechanism.

A series of experiments on alkali metal nanocontacts ${ }^{7,8,9}$ identified electron-shell effects as another key mechanism influencing nanowire stability. Energetically-favorable structures were revealed as peaks in conductance histograms, periodic in the nanowire radius, analogous to the electron-shell structure previously observed in metal clusters. ${ }^{15}$ A supershell structure was also observed, ${ }^{8}$ in the form of a periodic modulation of the peak heights. Recently, electron-shell structure has also been observed for the noble metals gold, ${ }^{10,11}$ copper, ${ }^{12}$ and silver. ${ }^{12}$

A theoretical analysis using the nanoscale free-electron model $^{16,17}$ (NFEM) found that nanowire stability is determined by the competition of these two key factors, surface tension and electron-shell effects. Both linear ${ }^{13,18,19}$ and nonlinear ${ }^{20,21}$ stability of axially sym- metric nanowires were investigated. It was found that the surface-tension driven instability can be completely suppressed in the vicinity of certain "magic radii."

It is well known in the physics of crystals and molecules that a Jahn-Teller deformation breaking the symmetry of the system can be energetically favorable. In metal clusters, Jahn-Teller deformations are also very common, ${ }^{22,23}$ and most of the observed structures show a broken spherical symmetry. By analogy, it is natural to assume that for nanowires, too, a breaking of axial symmetry can be energetically favorable, and lead to more stable deformed geometries.

Recently, as a first step towards a stability analysis of wires with a more general cross section, elliptical wires were examined within the NFEM. ${ }^{24}$ The sequence of stable cylindrical and elliptical nanowires allows for a consistent interpretation of experimental conductance histograms for alkali metals, including both the electronic shell and supershell structures. ${ }^{25}$

Note that while the experimental manifestations of electron-shell structure are similar in metal clusters and nanowires, the Jahn-Teller effect plays out quite differently in these two systems. The fundamental difference is that surface effects tend to stabilize clusters, while they are the source of the Rayleigh instability in nanowires. ${ }^{18}$ Therefore, Jahn-Teller deformations of clusters are very common and typically rather small, ${ }^{22,23}$ while they only occur for a minority of stable nanowires and can be rather large.

Cylindrical and elliptical nanowires are special, in the sense that the classical electron dynamics in these structures is integrable. An open question is whether integrability per se plays a special role in nanowire stability. On the one hand, the shell effect is enhanced in integrable systems, ${ }^{26}$ which argues in favor of integrability. On the other hand, the Jahn-Teller effect is driven by the lifting of degeneracy due to symmetry breaking, and the degeneracy of states with angular momentum $\pm \mu \hbar$ about the axis of symmetry is broken to leading order by a perturbation of the radius $\delta r(\phi) \propto \cos (2 \mu \phi)$, where $\phi$ is the az- 
imuthal angle, which renders the dynamics chaotic. Furthermore, for the case $\mu=1$ (quadrupolar cross section), the surface-energy cost of the perturbation is somewhat less than that of an elliptical deformation with the same aspect ratio.

In this article, we perform a general linear stability analysis for straight metal nanowires, including arbitrarily shaped, non-integrable cross sections. By considering all symmetry-breaking deformations, it is rigorously shown that the cylinder is the only generically stable structure. We derive the complete stability diagram for cylinders, and discuss why axial symmetry is special - in the sense that $75 \%$ of the experimentally observed alkali metal nanowires are indeed cylindrical, and that proportion goes up with increasing conductance.

Nevertheless, a wide range of structures with broken axial symmetry is found at low conductance values, most of which show a reduced relative stability compared to the axially symmetric ones. We examine wires with quadrupolar, hexapolar and octupolar cross sections, and compare these non-integrable shapes to the previous results on elliptical cross sections. Their relative stability and their degree of deformation are material-dependent, and we examine these properties for the alkali and noble metals.

This paper is organized as follows: The assumptions and features of the nanoscale free-electron model are summarized in Sec. II, followed by the presentation of the linear stability analysis in Sec. III. Cylindrical wires are examined in Sec. IV, whereas Sec. V considers nonintegrable cross sections. The material dependence of our results is discussed in Sec. VI. Finally, we summarize and discuss our results in Sec. VII. The Appendix provides additional technical details of our calculations.

\section{NANOSCALE FREE ELECTRON MODEL}

Guided by the importance of conduction electrons in the cohesion of metals, and by the success of the jellium model in describing metal clusters, ${ }^{15,27}$ the nanoscale free-electron model ${ }^{16,17}$ replaces the metal ions by a uniform, positively charged background that provides a confining potential for the electrons. The electron motion is free along the wire, and confined in the transverse directions. Due to the excellent screening ${ }^{28,29}$ in metal wires with $G>G_{0}$, where $G_{0}=2 e^{2} / h$ is the conductance quantum, electron-electron interactions can in most cases be neglected. The surface properties of various metals can be fit by using appropriate surface boundary conditions. ${ }^{24,30}$

The NFEM is especially suitable for alkali metals, but is also adequate to describe shell effects due to the conduction-band $s$-electrons in other monovalent metals, such as noble metals, and in particular, gold. The experimental observation of a crossover from atomic-shell to electron-shell effects with decreasing radius in both metal clusters ${ }^{31}$ and nanowires ${ }^{9}$ justifies a posteriori the use of the NFEM in the later regime.

Since a nanowire connecting two macroscopic electrodes is an open quantum system, the Schrödinger equation is most naturally formulated as a scattering problem. The fundamental theoretical quantity is the scattering matrix $S(E)$ connecting incoming and outgoing asymptotic states of conduction electrons in the electrodes. Thermodynamic properties can be expressed in terms of the scattering matrix through the electronic density of states $^{32}$

$$
D(E)=\frac{1}{2 \pi i} \operatorname{Tr}\left\{S^{\dagger}(E) \frac{\partial S}{\partial E}-\frac{\partial S^{\dagger}}{\partial E} S(E)\right\},
$$

from which the relevant thermodynamic potential for an open system, namely the grand canonical potential $\Omega$, is obtained as

$$
\Omega=-\frac{1}{\beta} \int d E D(E) \ln \left[1+e^{-\beta(E-\mu)}\right] .
$$

Here $\beta=\left(k_{B} T\right)^{-1}$ is the inverse temperature and $\mu$ is the electron chemical potential, specified by the macroscopic electrodes. Eqs. (1) and (2) include a factor of 2 for spin degeneracy.

Any extensive thermodynamic quantity can be expressed as the sum of a Weyl expansion, which depends on geometrical quantities such as the system volume $\mathcal{V}$, surface area $\mathcal{S}$, and integrated mean curvature $\mathcal{C}$, and an oscillatory shell-correction due to quantum-size effects. ${ }^{26}$ In particular, the grand canonical potential (2) can be written as

$$
\Omega=-\omega \mathcal{V}+\sigma_{s} \mathcal{S}-\gamma_{s} \mathcal{C}+\delta \Omega,
$$

where the energy density $-\omega$, surface tension coefficient $\sigma_{s}$, and curvature energy $-\gamma_{s}$ are, in general, materialand temperature-dependent coefficients. On the other hand, the shell correction $\delta \Omega$ can be shown, based on very general arguments, ${ }^{29,33,34}$ to be a single-particle effect, which is well described by the NFEM.

The ionic degrees of freedom in the NFEM are modeled as an incompressible fluid. ${ }^{13,20,21}$ This takes into account, to lowest order, the hard-core repulsion of the core electrons as well as the exchange energy of the conduction electrons. Any atom-conserving deformation of the structure is therefore subject to a constraint of the form

$$
\mathcal{N} \equiv k_{F}^{3} \mathcal{V}-\eta k_{F}^{2} \mathcal{S}+\xi k_{F} \mathcal{C}=\text { const },
$$

where $k_{F}$ is the Fermi wavevector. The parameters $\eta$ and $\xi$ can be adjusted so as to fix the values of the effective surface tension and curvature energy in the free-electron model to the material-specific values, as discussed in detail in Sec. VI. However, the shell correction $\delta \Omega$ is (to leading order) independent ${ }^{24,34}$ of $\eta$ and $\xi$.

In this article, the shell correction $\delta \Omega$ is calculated quantum-mechanically, from Eqs. (1) and (2). Nonetheless, in interpreting our quantum-mechanical results, it is useful to appeal to semiclassical arguments, ${ }^{26,35}$ according to which the shell correction can be computed by a 
Gutzwiller-type sum ${ }^{36}$ over classical periodic orbits, that takes the form of an integral over the length $L$ of the nanowire ${ }^{20}$

$$
\delta \Omega=\frac{E_{F}}{\lambda_{F}} \int_{0}^{L} d z \sum_{p o} A_{p o}(z, T) \cos \left(k_{F} L_{p o}(z)+\theta_{p o}\right),
$$

where $E_{F}$ and $\lambda_{F}$ are the Fermi energy and Fermi wavelength, respectively. Here the sum includes all classical periodic orbits lying in the plane of the wire's cross section, $L_{p o}$ is the orbit length, and $\theta_{p o}$ is an orbitdependent phase. Semiclassically, ${ }^{26}$ the temperature dependence of $\delta \Omega$ appears only in the dimensionless amplitude $A_{p o}$, which also depends on the stability, symmetry, and length of the periodic orbit. The orbit amplitude has the form ${ }^{26}$

$$
A_{p o} \sim\left(k_{F} L_{p o}\right)^{-\nu_{p o}} \frac{\tau_{p o}}{\sinh \tau_{p o}}, \quad \tau_{p o} \equiv \frac{\pi k_{F} L_{p o} T}{2 T_{F}},
$$

where $T_{F}=E_{F} / k_{B}$ is the Fermi temperature, and $\nu_{p o}=1$ for a continuous family of degenerate orbits, which arise when the cross section has the form of an integrable billiard, such as a disk or an ellipse, and $\nu_{p o}=3 / 2$ for an isolated orbit in a wire with a cross section whose dynamics is fully chaotic (see Ref. 37 for details).

It is useful to define the r.m.s radius $\rho$ of the cross section, which is related to the cross-sectional area by $\mathcal{A}=$ $\pi \rho^{2}$. Then $L_{p o} \propto \rho$, so that $\delta \Omega \sim \rho^{-1}$ for an integrable cross section, and decays exponentially for temperatures exceeding the radius-dependent characteristic temperature $T_{\rho} \equiv T_{F} / k_{F} \rho$. As shown below, these semiclassical estimates of the temperature- and size-dependence of $\delta \Omega$ are in accord with our quantum-mechanical results.

\section{LINEAR STABILITY ANALYSIS}

Let us consider a wire with uniform cross section aligned along the $z$-axis. Its surface is given by the radius function $r=R(\varphi ; \rho, \boldsymbol{\Lambda})$ in cylindrical coordinates $r, \varphi, z$, where the parameters $\{\rho, \boldsymbol{\Lambda}\}$ describe the cross section geometry, chosen such that the cross-sectional area is given by $\mathcal{A}=\pi \rho^{2}$, whereas the shape is determined by a set of dimensionless parameters, composing the vector $\boldsymbol{\Lambda}$. Without loss of generality, we can associate $\boldsymbol{\Lambda}=0$ with a cylindrical wire.

A small $z$-dependent perturbation of a wire of length $L$ and initial cross section $(\bar{\rho}, \overline{\boldsymbol{\Lambda}})$ can be written in terms of a Fourier series as

$$
\begin{gathered}
\rho(z)=\bar{\rho}+\epsilon \delta \rho(z)=\bar{\rho}+\epsilon \sum_{q} \rho_{q} e^{i q z} \\
\boldsymbol{\Lambda}(z)=\overline{\boldsymbol{\Lambda}}+\epsilon \delta \boldsymbol{\Lambda}(z)=\overline{\boldsymbol{\Lambda}}+\epsilon \sum_{q} \boldsymbol{\Lambda}_{q} e^{i q z}
\end{gathered}
$$

where the dimensionless small parameter $\epsilon$ sets the size of the perturbation. Assuming periodic boundary conditions, the perturbation wave vectors $q$ must be integer multiples of $2 \pi / L$. In order to ensure that $\rho(z)$ and $\boldsymbol{\Lambda}(z)$ are real, we have $\rho_{-q}=\rho_{q}^{*}$ and $\boldsymbol{\Lambda}_{-q}=\boldsymbol{\Lambda}_{q}^{*}$.

The structural stability of metal nanowires is governed by the response to long-wavelength perturbations, ${ }^{13,17,18,24}$ while the response to shortwavelength perturbations controls surface quantum fluctuations in long wires. ${ }^{19,38}$ Since we consider free electrons, the stationary Schrödinger equation is given by $-\triangle \Psi=\left(2 m_{e} E / \hbar^{2}\right) \Psi$, where $\triangle$ is the Laplace operator in cylindrical coordinates. In the long-wavelength limit, the Schrödinger equation may be solved in the adiabatic approximation, for which transverse and longitudinal motions decouple. Therefore, we use the ansatz $\Psi(r, \varphi, z)=\chi(r, \varphi ; z) \Phi(z)$, and neglect all $z$-derivatives of the transverse wavefunction $\chi$, so that the Schrödinger equation becomes

$$
\begin{aligned}
\left(\frac{\partial^{2}}{\partial r^{2}}+\frac{1}{r} \frac{\partial}{\partial r}+\frac{1}{r^{2}} \frac{\partial^{2}}{\partial \varphi^{2}}+\frac{2 m_{e}}{\hbar^{2}} E_{n}(z)\right) \chi_{n}(r, \varphi ; z) & =0,(8) \\
\left(\frac{\partial^{2}}{\partial z^{2}}+\frac{2 m_{e}}{\hbar^{2}}\left(E-E_{n}(z)\right)\right) \Phi_{n}(z) & =0
\end{aligned}
$$

First, the transverse problem (8) is solved at fixed $z$ in order to determine the transverse eigenenergies $E_{n}(z)$. With our choice of cross section parametrization, their dependence on geometry can be written as

$$
E_{n}(\rho, \boldsymbol{\Lambda})=\frac{\hbar^{2}}{2 m_{e}}\left(\frac{\gamma_{n}(\boldsymbol{\Lambda})}{\rho}\right)^{2},
$$

where the function $\gamma_{n}(\boldsymbol{\Lambda})$ remains to be determined. For an axisymmetric wire, the $\gamma_{n}$ are given by the roots of the Bessel functions, whereas for an elliptical cross section, the $\gamma_{n}$ are given by the roots of the modified Mathieu functions. ${ }^{24}$ In general, the shape-dependent $\gamma_{n}(\boldsymbol{\Lambda})$ are determined numerically (see Appendix for details).

We are then left with a series of effective onedimensional scattering problems (Eq. 9) for the longitudinal wave functions $\Phi_{n}(z)$, in which the transverse eigenenergies $E_{n}(\rho(z), \boldsymbol{\Lambda}(z))$ act as additional potentials for the motion along the wire. These scattering problems can be solved using the WKB approximation ${ }^{16}$ : The grand canonical potential is given by

$$
\Omega[T ; \rho, \boldsymbol{\Lambda}]=\int_{0}^{\infty} d E\left(-\frac{\partial f}{\partial E}\right) \Xi[E ; \rho, \boldsymbol{\Lambda}],
$$

where $f=\left(1+\exp \left[(E-\mu) / k_{B} T\right]\right)^{-1}$ is the Fermi function at temperature $T$ and chemical potential $\mu \approx E_{F}$, and $\Xi$ is given by

$$
\Xi[E ; \rho, \boldsymbol{\Lambda}]=-\frac{8 E_{F}}{3 \lambda_{F}} \int_{0}^{L} \mathrm{~d} z \sum_{n}\left(\frac{E-E_{n}(\rho, \boldsymbol{\Lambda})}{E_{F}}\right)^{3 / 2} .
$$

The sum runs over all open channels $n$, for which $E_{n}(\rho, \boldsymbol{\Lambda})<E$.

The energetic cost of a small deformation of the wire can be calculated by expanding Eq. (11) as a series in the parameter $\epsilon$,

$$
\Omega=\Omega^{(0)}+\epsilon \Omega^{(1)}+\epsilon^{2} \Omega^{(2)}+\mathcal{O}\left(\epsilon^{3}\right) .
$$


A nanowire with initial cross section $(\bar{\rho}, \bar{\Lambda})$ is energetically stable at temperature $T$ if and only if $\Omega^{(1)}(\bar{\rho}, \bar{\Lambda})=$ 0 and $\Omega^{(2)}(\bar{\rho}, \overline{\boldsymbol{\Lambda}})>0$ for every possible deformation $(\delta \rho, \delta \boldsymbol{\Lambda})$ satisfying the constraint (4).

A straightforward expansion of Eq. (11) at $T=0$ yields

$$
\begin{aligned}
& \frac{\Omega^{(1)}}{L / \lambda_{F}}=4 \sum_{n} \sqrt{\frac{E_{F}-\bar{E}_{n}}{E_{F}}}\left(\boldsymbol{\Lambda}_{0} \cdot \nabla_{\boldsymbol{\Lambda}} \bar{E}_{n}-2 \bar{E}_{n} \frac{\rho_{0}}{\bar{\rho}}\right), \\
& \frac{\Omega^{(2)}}{L / \lambda_{F}}=E_{F} \sum_{q}\left(\begin{array}{c}
\rho_{q} / \bar{\rho} \\
\boldsymbol{\Lambda}_{q}
\end{array}\right)^{\dagger}\left(\begin{array}{cc}
A_{\rho \rho} & A_{\rho \boldsymbol{\Lambda}} \\
A_{\boldsymbol{\Lambda} \rho} & A_{\boldsymbol{\Lambda} \boldsymbol{\Lambda}}
\end{array}\right)\left(\begin{array}{c}
\rho_{q} / \bar{\rho} \\
\boldsymbol{\Lambda}_{q}
\end{array}\right),
\end{aligned}
$$

where the elements of the matrix $A$ in Eq. (15) are given by

$$
\begin{aligned}
& A_{\rho \rho}=\sum_{n} \frac{4 \bar{E}_{n}}{E_{F}^{3 / 2}}\left[3 \sqrt{E_{F}-\bar{E}_{n}}-\frac{\bar{E}_{n}}{\sqrt{E_{F}-\bar{E}_{n}}}\right], \\
& A_{\boldsymbol{\Lambda} \rho}=-\sum_{n} \frac{4 \bar{E}_{n}^{\prime}}{E_{F}^{3 / 2}}\left[\sqrt{E_{F}-\bar{E}_{n}}-\frac{\bar{E}_{n}}{2 \sqrt{E_{F}-\bar{E}_{n}}}\right], \\
& A_{\boldsymbol{\Lambda} \boldsymbol{\Lambda}}=\sum_{n} \frac{1}{E_{F}^{3 / 2}}\left[2 \bar{E}_{n}^{\prime \prime} \sqrt{E_{F}-\bar{E}_{n}}-\frac{\bar{E}_{n}^{\prime} \cdot\left(\bar{E}_{n}^{\prime}\right)^{\dagger}}{\sqrt{E_{F}-\bar{E}_{n}}}\right] .
\end{aligned}
$$

Here $\bar{E}_{n}=E_{n}(\bar{\rho}, \overline{\boldsymbol{\Lambda}}), \quad \bar{E}_{n}^{\prime}=\left.\nabla_{\boldsymbol{\Lambda}} E_{n}\right|_{\bar{\rho}, \bar{\Lambda}}$ denotes the gradient of $E_{n}$ with respect to $\boldsymbol{\Lambda}$, and $\left(\bar{E}_{n}^{\prime \prime}\right)_{i j}=$ $\partial^{2} E_{n} /\left.\partial \Lambda_{i} \partial \Lambda_{j}\right|_{\bar{\rho}, \bar{\Lambda}}$ is the matrix of second derivatives, all evaluated at $(\bar{\rho}, \bar{\Lambda})$.

The number of independent Fourier coefficients in Eq. (7) is restricted through the constraint (4) on allowed deformations. Hence, after evaluating the change of the geometric quantities $\mathcal{V}, \mathcal{S}$, and $\mathcal{C}$ due to the deformation, we can use Eq. (4) to express $\rho_{0}$ in terms of the other Fourier coefficients. This yields an expansion $\rho_{0}=\rho_{0}^{(0)}+$ $\epsilon \rho_{0}^{(1)}+\mathcal{O}\left(\epsilon^{2}\right)$, with

$$
\begin{aligned}
& \frac{\rho_{0}^{(0)}}{\bar{\rho}}=\frac{\eta}{k_{F} \bar{\rho}-\eta \bar{P}} \bar{P}^{\prime} \cdot \boldsymbol{\Lambda}_{0}, \\
& \frac{\rho_{0}^{(1)}}{\bar{\rho}}=\frac{1}{2\left(k_{F} \bar{\rho}-\eta \bar{P}\right)} \sum_{q}\left(\begin{array}{c}
\rho_{q} / \bar{\rho} \\
\boldsymbol{\Lambda}_{q}
\end{array}\right)^{\dagger}\left(\begin{array}{cc}
-k_{F} \bar{\rho} & \eta \bar{P}^{\prime} \\
\eta\left(\bar{P}^{\prime}\right)^{T} & \eta \bar{P}^{\prime \prime}
\end{array}\right)\left(\begin{array}{c}
\rho_{q} / \bar{\rho} \\
\boldsymbol{\Lambda}_{q}
\end{array}\right),
\end{aligned}
$$

where the function $\bar{P}=P(\overline{\mathbf{\Lambda}})$ is related to the perimeter $\mathcal{P}$ by $\mathcal{P}(\bar{\rho}, \bar{\Lambda})=2 \pi \bar{\rho} \bar{P}$, the first derivative $\bar{P}^{\prime}=\left.\nabla_{\boldsymbol{\Lambda}} P\right|_{\bar{\Lambda}}$ is a vector, and the second derivative $\bar{P}^{\prime \prime}=\partial^{2} P /\left.\partial \Lambda_{i} \partial \Lambda_{j}\right|_{\bar{\Lambda}}$ is a matrix. Note that in the long-wavelength limit considered here, Eq. (17) is independent of the parameter $\xi$ appearing in the constraint (4), so that our results are independent of the value of $\xi$. Inserting the expansion for $\rho_{0}$ in Eq. (14), the first-order change of the energy under a constant $\mathcal{N}$ perturbation is given by

$$
\begin{aligned}
\left.\frac{\lambda_{F}}{L} \Omega^{(1)}\right|_{\mathcal{N}}= & \frac{4}{\sqrt{E_{F}}} \sum_{n} \sqrt{E_{F}-\bar{E}_{n}} \\
& \times\left(\bar{E}_{n}^{\prime}-\frac{2 \eta \bar{E}_{n}}{k_{F} \bar{\rho}-\eta \bar{P}} \bar{P}^{\prime}\right) \cdot \boldsymbol{\Lambda}_{0},
\end{aligned}
$$

and the second-order term is given by Eq. (15), with the matrix $A$ replaced by

$$
\begin{aligned}
& \tilde{A}_{\rho \rho}=A_{\rho \rho}+\frac{4 k_{F} \bar{\rho}}{k_{F} \bar{\rho}-\eta \bar{P}} \sum_{n} \frac{\bar{E}_{n} \sqrt{E_{F}-\bar{E}_{n}}}{E_{F}^{3 / 2}} \\
& \tilde{A}_{\boldsymbol{\Lambda} \rho}=A_{\boldsymbol{\Lambda} \rho}-\frac{4 \eta P^{\prime}}{k_{F} \bar{\rho}-\eta \bar{P}} \sum_{n} \sqrt{\frac{E_{F}-\bar{E}_{n}}{E_{F}}} \\
& \tilde{A}_{\boldsymbol{\Lambda} \Lambda}=A_{\boldsymbol{\Lambda} \Lambda}-\frac{4 \eta \bar{P}^{\prime \prime}}{k_{F} \bar{\rho}-\eta \bar{P}} \sum_{n} \sqrt{\frac{E_{F}-\bar{E}_{n}}{E_{F}}}
\end{aligned}
$$

The stability condition, $\left.\Omega^{(2)}(\bar{\rho}, \bar{\Lambda})\right|_{\mathcal{N}}>0$, requires that the stability matrix $\tilde{A}$ be positive definite. The results at finite temperature are obtained in a similar fashion, by integrating Eq. (11) numerically.

\section{GENERAL STABILITY OF CYLINDERS}

As a first application of the method presented in the previous sections, we derive the complete stability diagram for cylinders, i.e., we determine the radii of cylindrical wires that are linearly stable with respect to arbitrary small, long-wavelength deformations. We choose the following general form of the radius function describing the surface of the wire,

$$
R(\varphi)=\rho\left(\sqrt{1-\sum_{m} \frac{\lambda_{m}^{2}}{2}}+\sum_{m} \lambda_{m} \cos \left[m\left(\varphi-\varphi_{m}\right)\right]\right) .
$$

The sum runs over a set $\mathbb{M}$ of positive integer values, and the deformation parameters $\lambda_{m}$ governing deviations from axial symmetry are such that $\sum_{m} \lambda_{m}^{2}<2$. Note that the dipole deformation $(m=1)$ corresponds, in leading order, to a simple translation, plus higher-order multipole deformations. Therefore we can restrict our analysis to $m>1$.

At first sight, considering arbitrary deformations, and therefore theoretically an infinite number of perturbation parameters $\left\{\lambda_{m}, \varphi_{m}\right\}$ seems a formidable task. Fortunately, we find that the stability matrix $\tilde{A}$ for a cylinder is diagonal, and therefore the different Fourier contributions of the deformation decouple.

This can be seen within perturbation theory for small deviations from axial symmetry, derived in the Appendix: for energy levels $n$ that are non-degenerate at $\boldsymbol{\Lambda}=0$, i.e. levels with orbital quantum number $\mu_{n}=0$, we obtain

$$
\frac{\partial E_{n}(0)}{\partial \Lambda_{i}}=0 \quad \text { and } \quad \frac{\partial^{2} E_{n}(0)}{\partial \Lambda_{i} \partial \Lambda_{j}} \propto \delta_{i j},
$$

whereas for two energy levels $n$ and $n^{\prime}$ that are degenerate at $\boldsymbol{\Lambda}=0$, i.e. those for which $\mu_{n}=-\mu_{n^{\prime}} \neq 0$, we get

$$
\frac{\partial E_{n}(0)}{\partial \Lambda_{i}}=-\frac{\partial E_{n^{\prime}}(0)}{\partial \Lambda_{i}},
$$




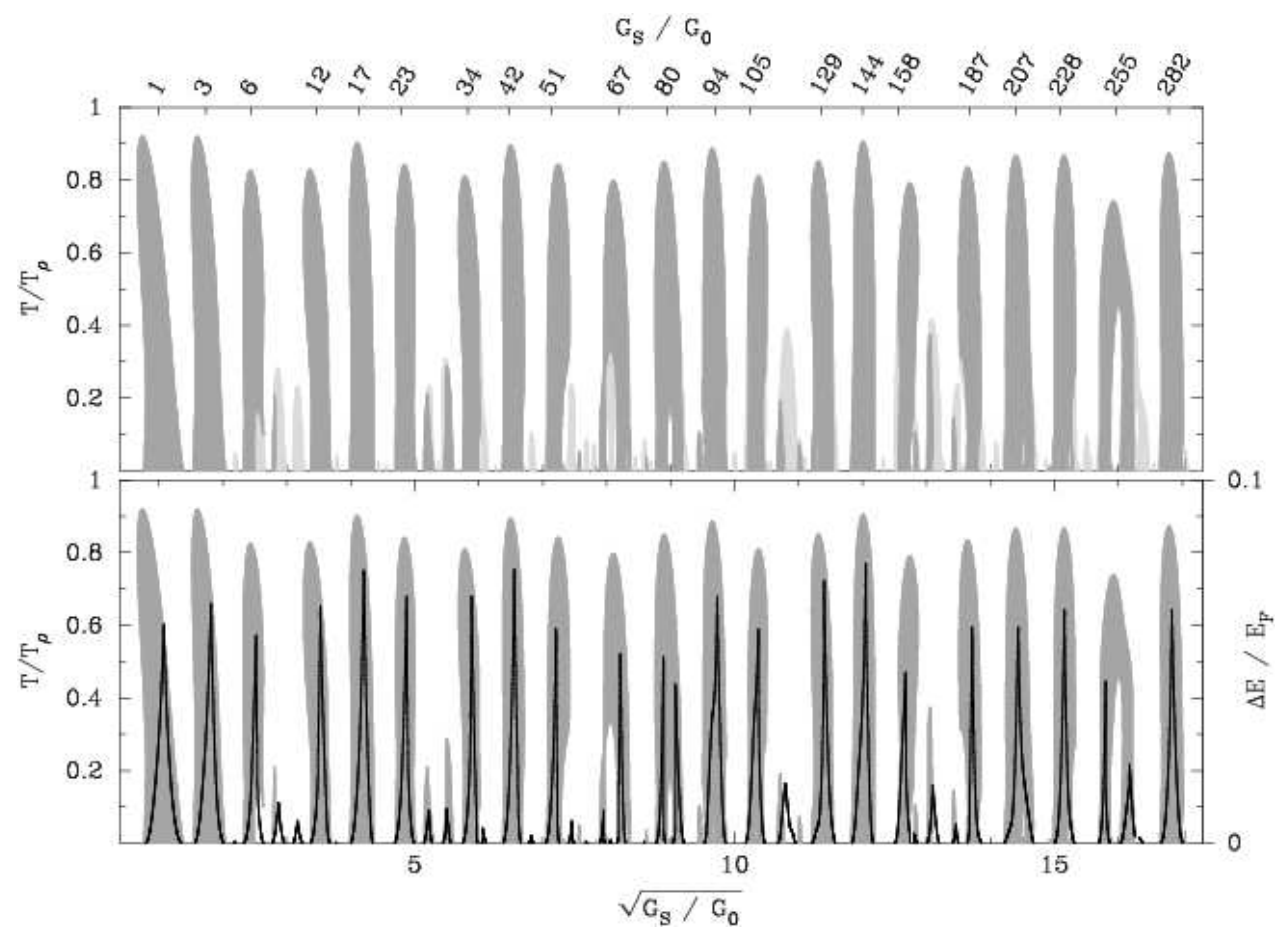

FIG. 1: Stability of metal nanocylinders versus electrical conductance and temperature. Dark gray areas indicate stability with respect to arbitrary small deformations. Temperature is displayed in units of $T_{\rho}=$ $T_{F} / k_{F} \rho$ (see text). The surface tension was taken as $0.22 \mathrm{~N} / \mathrm{m}$, corresponding to $\mathrm{Na}^{39}$ For comparison, the upper panel also shows wires that are stable towards axisymmetric deformations only (light gray areas), but which are unstable when allowing symmetry breaking deformations. The lower panel includes a plot of the activation energy $\Delta E$ (solid line), reproduced from Ref. 21, which determines the nanowire lifetime $\tau=\tau_{0} \exp \left(\Delta E / k_{B} T\right)$.

and

$$
\frac{\partial^{2} E_{n}(0)}{\partial \Lambda_{i} \partial \Lambda_{j}}+\frac{\partial^{2} E_{n^{\prime}}(0)}{\partial \Lambda_{i} \partial \Lambda_{j}} \propto \delta_{i j} .
$$

Since the matrix $A$, given by Eq. (16), includes a sum over all open channels, the relations (21-23) imply that $A$ is diagonal. Furthermore, one can straightforwardly show, by expanding the perimeter function $P$ in a series in $\Lambda$, that $\bar{P}^{\prime}=\left.\nabla_{\boldsymbol{\Lambda}} P\right|_{\boldsymbol{\Lambda}=0}=0$ and the matrix $\bar{P}^{\prime \prime}=\partial^{2} P /\left.\partial \Lambda_{i} \partial \Lambda_{j}\right|_{\Lambda=0}$ is diagonal as well. Therefore, we conclude that the stability matrix $\tilde{A}$, including the corrections from the constraint (4), is diagonal. This also shows that the first order correction (18) to $\Omega$ is identically zero for cylinders. This result implies a great simplification of the problem, since it allows to determine the stability of cylindrical wires with respect to arbitrary deformations through the study of a set of pure $m$-deformations, i.e. deformations as given by Eq. (20) with only one non-zero $\lambda_{m}$.

Fig. 1 shows the stable cylindrical wires in dark gray as a function of temperature. The surface tension was fixed at the value ${ }^{39}$ for $\mathrm{Na}, \sigma_{s}=0.22 \mathrm{~N} / \mathrm{m}$ (i.e. $\eta=1.1$ ). The temperature is given in units of the characteristic temperature $T_{\rho}=T_{F} / k_{F} \rho$, reflecting the temperature dependence of the shell correction (5) to the wire energy: Since stability is determined by a competition between the surface and shell contributions, ${ }^{17,18}$ the maximum temperature of linear stability scales with $T_{\rho}$, the surface contribution being only weakly dependent on temperature. The $x$-axis is given by the corrected Sharvin conductance

$$
G_{S}=G_{0}\left(\frac{k_{F}^{2} \rho^{2}}{4}-\frac{k_{F} \mathcal{P}}{4 \pi}+\frac{1}{6}\right),
$$

where $\mathcal{P}$ is the perimeter of the cross section.

The stability diagram was obtained by intersecting a set of individual stability diagrams allowing $\cos (m \varphi)$ deformations with $m=2, \ldots, 8$. Multipolar deformations become increasingly costly with increasing $m$, their surface energy scaling as $\rho m^{2}$, and we find that including $m \geq 6$ deformations does not further modify the final result.

Figure 1 confirms the extraordinary stability of a set of wires with so called "magic radii," which were identified in previous studies of stability under axisymmetric perturbations alone. ${ }^{13,18,19,21}$ They exhibit conductance values $G / G_{0}=1,3,6,12,17,23,34,42,51, \ldots$ However, some wires that are barely stable when considering only axisymmetric perturbations, ${ }^{13,18,19}$ e.g., $G / G_{0}=5$, $10,14, \ldots$, shown in light gray in the top panel of Fig. 1 , are found to be unstable when allowing more general, symmetry-breaking deformations.

The heights of the dominant stability peaks in Fig. 1 exhibit a periodic modulation, with minima occurring near $G_{S} / G_{0}=9,29,59,117,170$, and 255 . The positions of the first four minima are in perfect agreement with the observed supershell structure in conductance histograms of alkali metal nanowires ${ }^{8}$ while we predict the next two minima of the series. The supershell structure is expected to continue at larger conductance, but may be obscured by atomic-shell effects in experiments. Interestingly, the nodes of the supershell structure, where the shell effect for a cylinder is suppressed, are precisely where the most stable deformed nanowires are predicted to occur (see Ref. 24 and the discussion below). Thus symmetry breaking distortions and the supershell effect are inextricably linked.

Linear stability is a necessary — but not a sufficient- 


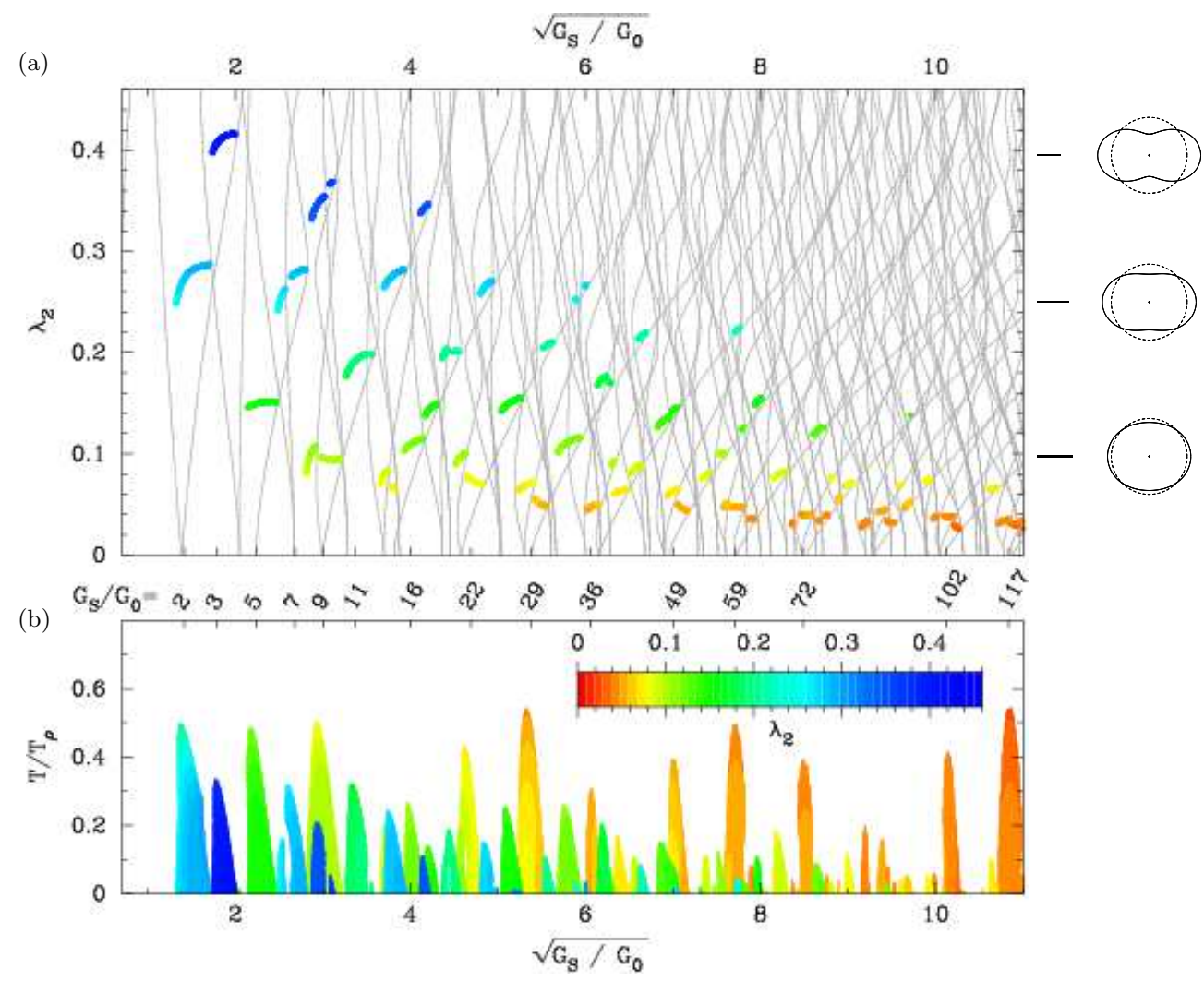

FIG. 2: (color online) (a) Linearly stable quadrupolar $\left(\lambda_{2}>0\right) \mathrm{Na}$ wires [thick lines] at temperature $T=0.03 T_{\rho}$, shown in the configuration space of the two parameters $\lambda_{2}$ and $\rho$ describing the quadrupolar shape, $R(\varphi)=$ $\rho\left(\sqrt{1-\lambda_{2}^{2} / 2}+\lambda_{2} \cos (2 \varphi)\right)$. $\rho$ is related to the Sharvin conductance by Eq. (24), which gives $\sqrt{G_{S} / G_{0}} \approx k_{F} \rho / 2$. The thin gray lines show the thresholds for the opening of new channels. Some geometries are shown on the right axis, illustrating their deviations from axial symmetry; (b) Stability diagram for quadrupolar $\mathrm{Na}$ wires. The gray(color) scale reflects the value of $\lambda_{2}$ for the stable wires.

condition for a nanostructure to be observed experimentally. The linearly stable nanocylinders revealed in the above analysis are in fact metastable structures, and an analysis of their lifetime has recently been carried out within an axisymmetric stochastic field theory. ${ }^{21}$ In the lower panel of Fig. 1, the zones of linear stability are superimposed on the activation energy $\Delta E$ (solid line), calculated in Ref. 21, which determines the nanowire lifetime $\tau$ through the Kramers formula $\tau=$ $\tau_{0} \exp \left(\Delta E / k_{B} T\right)$. As can be seen, there is a strong correlation between the size of the activation barriers and the height of the stable fingers in the linear stability analysis. This suggests that the linear stability analysis, with temperature expressed in units of $T_{\rho}=T_{F} / k_{F} \rho$, provides a good measure of the total stability of metal nanowires. In particular, the "universal" stability ${ }^{21}$ of the most stable cylinders is reproduced, wherein the absolute stability of the magic cylinders is essentially independent of radius (aside from the small supershell oscillations).

For a given material, the typical value $\Delta E$ for the magic cylinders is universal, i.e. it depends only on the surface tension ${ }^{21} \sigma_{s}$

$$
\Delta E \approx 0.6 \sqrt{\hbar^{2} \sigma_{s} / m_{e}}
$$

where $m_{e}$ is the electron effective mass, which is of order the free electron mass in simple metals. When comparing the absolute stability for different materials, it is not sufficient to simply compare the heights of the stable fingers in Fig. 1, which depend only logarithmically on the ratio $\sigma_{s} / E_{F} k_{F}^{2}$ through Eqs. (3) and (5); the $\sigma_{s}^{1 / 2}$ dependence of the activation barrier, which determines the lifetime, must be taken into account explicitly (see Sec. VI).

Since the activation barriers are calculated asymptotically for low temperatures ${ }^{21}$ they exhibit all of the fine structure of the low-temperature shell correction. This fine structure is also seen in the linear stability analysis at low temperature (see Fig. 1, lower panel). However, at higher temperatures, the fine structure in the shell potential is smoothed out, as described in Eqs. (5) and (6), and some of the separate low-temperature stability zones merge into arches at higher temperatures. Prominent examples of this phenomenon, the unusual thermodynamics of which is discussed in Ref. 13 , occur at $G_{S} / G_{0} \sim 67$, 80 , and 255 .

\section{BREAKING AXIAL SYMMETRY}

In a recent paper, ${ }^{24}$ the stability of wires with elliptic cross sections was determined. Elliptic deformations are special, in that the system remains integrable. Here we focus on wires with a $\cos (m \varphi)$-deformed cross section (i.e. having $m$-fold symmetry), a special case of Eq. (20) with only one non-zero $\lambda_{m}$, which renders the system nonintegrable. In the following, we will discuss quadrupolar deformations with $m=2$, which are the most energetically favorable of the multipole deformations, as well as the higher-order multipoles with $m \leq 6$. Deformations with higher $m$ cost significantly more surface energy, and 
thus yield fewer stable configurations. The stability analysis of wires with 5- and 6-fold symmetry reveals only a few barely stable configurations (besides the axisymmetric wires discussed above), whereas for $m>6$ no stable deformed wires were found at all in the temperature range considered.

Figure 2(a) shows the stable configurations [thick curves] for quadrupolar $\mathrm{Na}$ wires at temperature $T=$ $0.03 T_{\rho}$. These are determined by the intersection of the stationary curves, $\left.\Omega^{(1)}\left(\bar{\rho}, \bar{\lambda}_{2}\right)\right|_{\mathcal{N}}=0$, and the convex regions, $\left.\Omega^{(2)}\left(\bar{\rho}, \bar{\lambda}_{2}\right)\right|_{\mathcal{N}}>0$. Also shown are the thresholds to open new conducting channels, i.e., the energy eigenvalues of the two-dimensional billiard comprising the cross section of the wire. Note that the stable structures lie in the gaps of the spectrum of channel thresholds, as is the case for axisymmetric wires. ${ }^{18}$

Combining results for all temperatures yields the stability diagram shown in Fig. 2(b). Note that the maximum deformation of the stable structures decreases strongly with increasing conductance. Nanowires with highly-deformed cross sections are only stable at small conductance, unlike the magic cylinders, which are predicted to occur for arbitrarily large conductance (until the transition to crystalline structures at sufficiently large radius).

The most stable wires are found at the same conductance values, and with similar semi-axis ratios, as the stable elliptical wires, discussed in Ref. 24. This is not surprising, since the ellipse can be approximated to leading order by a quadrupole,

$$
\begin{aligned}
R_{\mathrm{ell}}(\varphi) \approx & \left(1-\kappa^{2}\right)+\left(2 \kappa+7 \kappa^{3}\right) \cos (2 \varphi)+3 \kappa^{2} \cos (4 \varphi) \\
& +5 \kappa^{3} \cos (6 \varphi)+\mathcal{O}\left(\kappa^{4}\right),
\end{aligned}
$$

where $\kappa \equiv \frac{1}{4}\left(\varepsilon^{2}-1\right) /\left(\varepsilon^{2}+1\right)$ is given in terms of the aspect ratio $\varepsilon$. The maximum temperature $T_{\max }$ up to which the wires are stable is in general of the same order as found for elliptical wires. Nevertheless, for the stable wire at $G=2 G_{0}, T_{\max }$ is 20 percent larger compared to the corresponding elliptical wire, indicating that a wire with two conducting channels will show a peanut-shaped cross section, rather than an elliptic one. Remarkably, this result, which was obtained by minimizing the electronic energy - and thus contains nothing of the atomic structure - is exactly what one would expect for a wire with two atoms in the cross section.

In order to shed light on the possible competition between integrable (elliptical) and non-integrable (quadrupolar) shapes, we analyzed the stability of wires with simultaneous $m=2$ and $m=4$ deformations, which can interpolate between elliptical and quadrupolar cross sections [cf. Eqs. (20) and (26)]. The results are summarized in Table I. For large deformations, the quadrupole is more stable, due to its lower surface energy, but no overall preference for a special geometry was found; stable wires with both positive and negative values of $\lambda_{4}$ exist. The values of $\lambda_{4}$ were found to be small, indicating that the addition of further deformations has little influence on the stability diagram. Remarkably, the wire
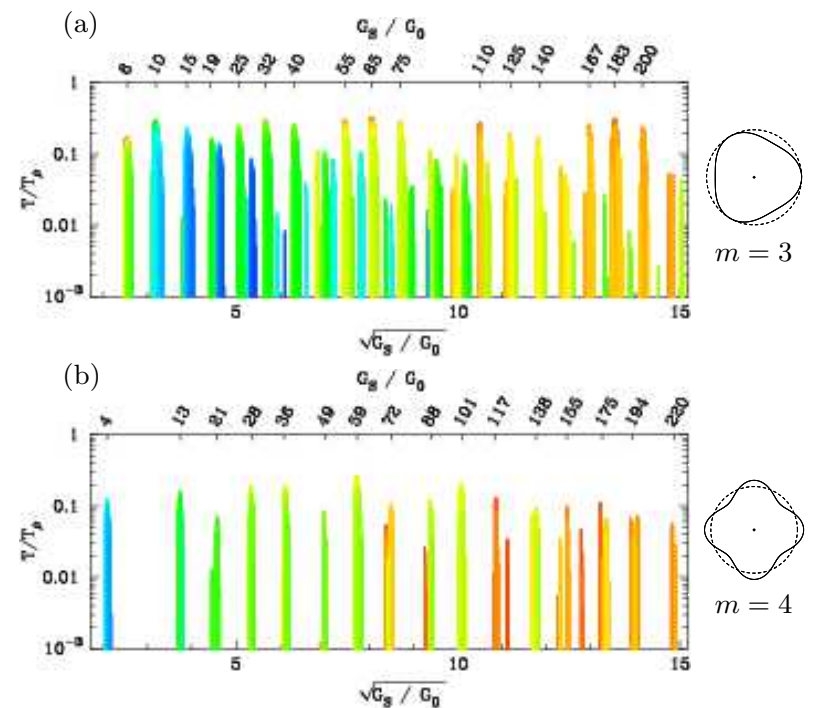

(c) $\quad G_{s} / G_{0}$

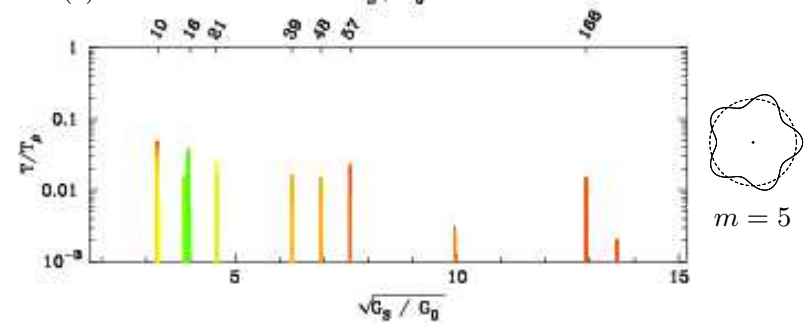

(d) $\quad \mathrm{G}_{\mathrm{g}} / \mathrm{G}_{\mathrm{o}}$

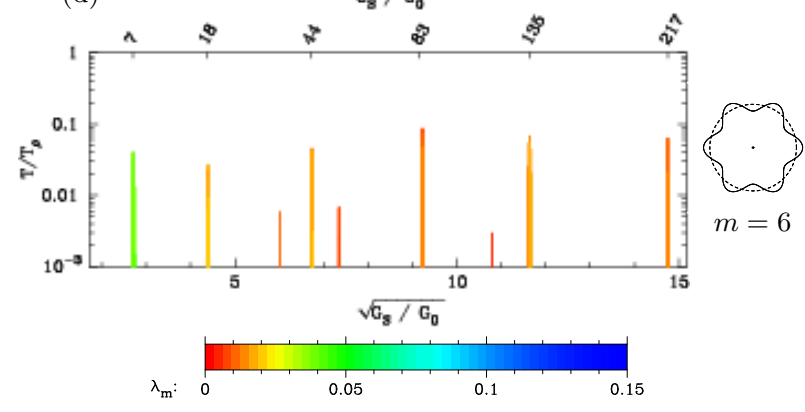

FIG. 3: (color online) Linear stability of $\cos (m \varphi)$-deformed wires for (a) $m=3$, (b) $m=4$, (c) $m=5$, and (d) $m=6$, as a function of temperature (in units of $T_{\rho}$ ). A logarithmic scale is used for the temperature since wires with $m \geq 4$ are much less stable than those for $m \leq 3$. A sketch of the cross section is shown on the right. The deformation parameter $\lambda_{m}$ is encoded via a gray(color) scale, common to all four diagrams. In the interest of clarity, only non-cylindrical wires (with $\lambda_{m}>0$ ) are shown. The surface tension was chosen to represent $\mathrm{Na}$.

with $G=2 G_{0}$ is even closer to two touching cylinders when 4 -fold deformations are added to the quadrupolar ones.

The linearly stable wires with 3-, 4-, 5-, and 6-fold symmetry are shown in Fig. 3 as a function of temperature. For clarity, the stable cylindrical wires are omitted, and a logarithmic scale is used for the temperature axis. The value of the deformation parameter $\lambda_{m}$ is encoded 


\begin{tabular}{|c||c|c|c|c||c|c|c||c|c|c|}
\hline \multicolumn{1}{|c||}{$G$} & \multicolumn{3}{c||}{ Ellipse } & \multicolumn{3}{c||}{$\lambda_{2} \cos (2 \varphi)$} & \multicolumn{3}{c|}{$\lambda_{2} \cos (2 \varphi)+\lambda_{4} \cos (4 \varphi)$} \\
{$\left[G_{0}\right]$} & $\varepsilon$ & $\lambda_{2}$ & $\lambda_{4}$ & $T_{\max } / T_{\rho}$ & $\varepsilon$ & $\lambda_{2}$ & $T_{\max } / T_{\rho}$ & $\lambda_{2}$ & $\lambda_{4}$ & $T_{\max } / T_{\rho}$ \\
\hline 2 & 1.65 & 0.24 & 0.0440 & 0.40 & 1.7 & 0.26 & 0.50 & 0.25 & -0.04 & 0.50 \\
5 & 1.32 & 0.14 & 0.0140 & 0.44 & 1.32 & 0.14 & 0.49 & 0.15 & $-0.03 /+0.08$ & $0.49 / 0.23$ \\
9 & 1.24 & 0.11 & 0.0086 & 0.50 & 1.22 & 0.10 & 0.50 & 0.10 & $0.03 /-0.03$ & $0.48 / 0.44$ \\
29 & 1.14 & 0.07 & 0.0032 & 0.54 & 1.13 & 0.06 & 0.54 & $0.07 / 0.05$ & $0.008 /-0.015$ & $0.44 / 0.44$ \\
59 & 1.09 & 0.04 & 0.0014 & 0.50 & 1.11 & 0.05 & 0.49 & 0.04 & -0.01 & 0.49 \\
72 & 1.08 & 0.04 & 0.0011 & 0.40 & 1.08 & 0.04 & 0.39 & 0.04 & 0.009 & 0.36 \\
\hline
\end{tabular}

TABLE I: Comparison of the most stable deformed wires with elliptic (columns 2-5), quadrupolar (columns 6-8), and more general (columns 9-11) cross sections. The first column gives the quantized conductance of the corresponding wire. For the elliptic and quadrupolar wires, both the aspect ratio and the value of the deformation parameters (obtained from Eq. (26) for the former) are given. The maximum temperature of stability $T_{\max }$ is given for each wire. The values for the elliptic wires are taken from Ref. 24. Note that multiple values indicate multiple stable wires with the same conductance. In all cases the surface tension was set to $0.22 \mathrm{~N} / \mathrm{m}$, corresponding to $\mathrm{Na}{ }^{39}$

through a gray(color) scale, which is common to all stability diagrams shown in this figure, but is different than the one of Fig. 2. Compared to the quadrupolar wires, the number of stable configurations, the maximum temperature of stability, and the size of the deformations involved, all decrease rapidly with increasing order $m$ of the deformation. For $m>6$, we no longer find stable geometries in the temperature range considered. All this reflects the increase in surface energy with increasing order $m$ of the deformation.

From Fig. 3(b), we can extract a series of stable wires with four-fold symmetry, for which the maximum deformation of $\lambda_{4} \sim 0.1$ is found at a conductance of $G=4 G_{0}$. Although this geometry is expected to be far less stable than the neighboring cylindrical and quadrupolar wires, it has nevertheless likely been observed as a shoulder in Na conductance histograms. ${ }^{25}$

\section{MATERIAL DEPENDENCE}

Within the NFEM there is only one parameter entering the calculation apart from the contact geometry: the Fermi energy $E_{F}$, which is material dependent and in general well known (see Tab. II). Nevertheless, we have seen in the previous section that the energy cost of a deformation due to surface and curvature energy, which can vary significantly for different materials, plays a crucial role in determining the stability of a nanowire. Using the NFEM a priori implies the macroscopic free energy density $\omega=2 E_{F} k_{F}^{3} / 15 \pi^{2}$, the macroscopic surface energy $\sigma_{s}=E_{F} k_{F}^{2} / 16 \pi$, and the macroscopic curvature energy $\gamma_{s}=2 E_{F} k_{F} / 9 \pi^{2}$. When drawing conclusions for metals having surface tensions and curvature energies that are rather different from these values, we have to think of an appropriate way to include these material-specific properties in our calculation.

Other authors have calculated the surface energy using a free-electron model with a rectangular confining potential of finite height, ${ }^{40,41}$ depending on the electron work function, without being able to reproduce the values found in experiments. The agreement does not improve even when using a self-consistent confining potential. ${ }^{41}$ When working with a free-electron model, contributions of correlation and exchange energy are not included, which are found to play an essential role for a correct treatment of the surface energy. A discussion of the calculation of the surface energy of a jellium metal beyond the free-electron model can be found in Ref. 42.

A convenient way of modeling the material properties without losing the pleasant features of the NFEM is via the implementation of the constraint (4) on the deformation, which interpolates between volume conservation $(\eta=\xi=0)$ and treating the semiclassical expectation value for the charge $N_{\text {weyl }}$ as an invariant $(\eta=3 \pi / 8$, $\xi=1)$.

Consider the grand canonical potential of a freeelectron gas confined within a given geometry by hardwall boundaries, as given by Eq. (3). The change in energy due to a deformation is

$$
\begin{aligned}
\Delta \Omega= & -\omega \Delta \mathcal{V}+\sigma_{s} \Delta \mathcal{S}-\gamma_{s} \Delta \mathcal{C}+\Delta[\delta \Omega] \\
= & -\frac{\omega}{k_{F}^{3}} \Delta \mathcal{N}+\left(\sigma_{s}-\frac{\omega}{k_{F}} \eta\right) \Delta \mathcal{S} \\
& -\left(\gamma_{s}-\frac{\omega}{k_{F}^{2}} \xi\right) \Delta \mathcal{C}+\Delta[\delta \Omega],
\end{aligned}
$$

where we have used the constraint (4) to eliminate $\mathcal{V}$. Now the prefactors of the change in surface $\Delta \mathcal{S}$ and the change in integrated mean curvature $\Delta \mathcal{C}$ can be identified as effective surface tension and curvature energy, respectively. They can be adjusted to fit the material properties by appropriate choice ${ }^{43}$ of the parameters $\eta$ and $\xi$ (see Tab. II). As noted previously, results in the long-wavelength limit are independent of $\xi$.

The stability diagrams discussed in the previous sections all represent sodium nanowires. Results for other s-orbital metals are similar in respect to the number of stable configurations and the conductance of the wires. On the other hand, the deviations from axial symmetry and the relative stability of Jahn-Teller deformed wires is sensitive to the material specific surface tension $\sigma_{s}$ 


\begin{tabular}{|l|c|c|c|c|c|c|}
\hline Element & $\mathrm{Li}$ & $\mathrm{Na}$ & $\mathrm{K}$ & $\mathrm{Cu}$ & $\mathrm{Ag}$ & $\mathrm{Au}$ \\
\hline$E_{F}[\mathrm{eV}]$ & 4.74 & 3.24 & 2.12 & 7.00 & 5.49 & 5.53 \\
$k_{F}\left[\mathrm{~nm}^{-1}\right]$ & 11.2 & 9.2 & 7.5 & 13.6 & 12.0 & 12.1 \\
\hline$\sigma_{s}\left[{\left.\mathrm{meV} / \AA^{2}\right]}^{2} 27.2\right.$ & 13.6 & 7.58 & 93.3 & 64.9 & 78.5 \\
$\sigma_{s}\left[E_{F} k_{F}^{2}\right]$ & 0.0046 & 0.0050 & 0.0064 & 0.0072 & 0.0082 & 0.0097 \\
$\eta$ & 1.135 & 1.105 & 1.001 & 0.939 & 0.866 & 0.755 \\
\hline$\gamma_{s}[\mathrm{meV} / \AA]$ & 62.0 & 24.6 & 14.9 & 119 & 96.4 & 161 \\
$\gamma_{s}\left[E_{F} k_{F}\right]$ & 0.0117 & 0.0082 & 0.0094 & 0.0125 & 0.0146 & 0.0240 \\
$\xi$ & 0.802 & 1.06 & 0.971 & 0.741 & 0.583 & -0.111 \\
\hline
\end{tabular}

TABLE II: Material parameters of several monovalent metals: Fermi energy $E_{F},{ }^{44}$ Fermi wavevector $k_{F},{ }^{44}$ surface tension $\sigma_{s}$, and curvature energy $\gamma_{s},{ }^{39}$ along with the corresponding values of $\eta$ and $\xi$.

and Fermi temperature $T_{F}$. The relative stability of the highly deformed wires decreases with increasing surface tension $\sigma_{s} / E_{F} k_{F}^{2}$, measured in intrinsic units, and this decrease becomes stronger with increasing order $m$ of the deformation. Therefore, for the simple metals under consideration (Tab. II), deformed Li wires have the highest, and $\mathrm{Au}$ wires have the lowest relative stability compared to cylinders of "magic radii."

However, concerning absolute stability, one must consider that the lifetime of a metastable nanowire also depends on the surface tension [cf. Eq. (25)]. Thus, although the deformed wires have reduced relative stability in the noble metals, this effect is compensated by the greater absolute stability of these materials.

In Fig. 4, the linearly stable quadrupolar wires for gold are shown. The vertical scale was adjusted according to the arguments discussed above, in order to allow a comparison with the absolute stability for $\mathrm{Na}$ wires, as shown in Fig. 2.

\section{SUMMARY AND DISCUSSION}

In this article, we have performed a complete linear stability analysis of metal nanowires within the NFEM. That is to say, we have examined the stability with re-

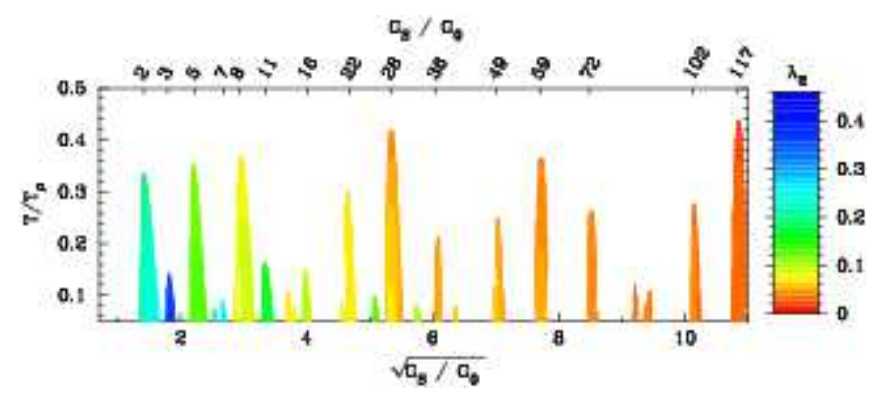

FIG. 4: Linearly stable Au wires with quadrupolar cross section. The deformation parameter $\lambda_{2}>0$ is coded via a color(gray) scale. spect to small, long-wavelength perturbations of metal nanostructures of arbitrary cross section, with translational symmetry along an axis perpendicular to the cross section. We have considered both cross sections for which the electron motion is integrable, namely circles and ellipses, and nonintegrable cross sections, described by a multipole expansion (20).

This general stability analysis confirms the central conclusions of previous analyses, which were limited to circular ${ }^{13,18,19}$ and elliptical cross sections. ${ }^{24,25}$ First, the existence of a sequence of "magic" cylindrical wires of exceptional stability, with electrical conductance $G / G_{0}=$ $1,3,6,12,17,23,34,42,51, \ldots$, was confirmed. Second, the existence of a series of very stable deformed wires, with two-fold symmetric cross sections, which occur at the nodes of the cylindrical shell structure, at $G / G_{0}=2$, $5,9,29,59, \ldots$, was verified. For the thinnest of these wires $\left(G / G_{0}=2,5\right)$, which have the largest deformations, the quadrupolar cross section was found to be more stable than the ellipse, while the cross sections of the thicker wires in the sequence were found to be essentially elliptical. In addition, a number of new stable structures with quadrupolar, hexapolar, and octupolar cross sections have been identified.

Perhaps the most surprising result of our general linear stability analysis is the special role played by cylinders. Roughly $75 \%$ of the principal structures observed in conductance histograms ${ }^{8}$ of alkali metals correspond to the magic cylinders. The remaining $25 \%$ are comprised of wires with elliptical/quadrupolar cross sections. Despite expanding the configuration space of allowed structures significantly in the present analysis, no new structures of comparable stability have been identified for the alkali or noble metals. The role of symmetry in the stability of metal nanowires is thus fundamentally different from the case of atomic nuclei ${ }^{45}$ or metal clusters, ${ }^{22,23}$ where the vast majority of stable structures have broken symmetry.

The crucial difference between the stability of metal nanowires and metal clusters is not the shell effect, which is similar in both cases, but rather the surface energy, which favors the sphere, but abhors the cylinder. ${ }^{14}$ In order to understand the unique stability of metal nanocylinders, in contrast to other wire structures, it is useful to consider the semiclassical size of the competing surface and shell contributions: From Eq. (3), the surface energy scales as

$$
\frac{\Omega_{s}}{E_{F} k_{F} L} \sim \mathcal{O}\left(k_{F} \rho\right),
$$

where $\rho$ is the r.m.s. radius of the wire. On the other hand, Eq. (5) implies that

$$
\frac{\delta \Omega}{E_{F} k_{F} L} \sim \mathcal{O}\left(k_{F} \rho\right)^{-\nu},
$$

where $\nu=1$ for circular or elliptical cross sections, and $3 / 2 \geq \nu>1$ for nonintegrable cross sections. For a typical metal nanowire in the domain of validity of the 
NFEM, $k_{F} \rho \sim 10$ (i.e., $G_{S} / G_{0} \sim 20$ ). Thus, the term $\Omega_{s}$ which gives rise to the Rayleigh instability ${ }^{14,18}$ is roughly two orders of magnitude larger than the shell correction $\delta \Omega$, which stabilizes the wire even in the best-case scenario of an integrable cross section! (The volume contribution to $\Omega$, though larger still than $\Omega_{s}$, does not play a role in deformations that conserve the number of atoms.) How then are we to understand the stability of metal nanowires?

Stability is not determined by the energy itself, but by the stationarity and convexity of the energy functional. Because $\delta \Omega$ is a rapidly oscillating function [cf. Eq. (5)], its derivatives are large in a semiclassical sense. ${ }^{46}$ The first and second variations of $\delta \Omega$ scale as

$$
\frac{\delta \Omega^{(1)}}{E_{F} k_{F} L} \sim \mathcal{O}\left(k_{F} \rho\right)^{1-\nu}, \quad \frac{\delta \Omega^{(2)}}{E_{F} k_{F} L} \sim \mathcal{O}\left(k_{F} \rho\right)^{2-\nu} .
$$

Thus, for the case of an integrable cross section $(\nu=1)$, $\delta \Omega^{(2)}$ is of the same semiclassical size as $\Omega_{s}^{(2)}$. However, $\delta \Omega^{(1)}$ is always semiclassically smaller than $\Omega_{s}^{(1)}$. The stationarity condition $\Omega^{(1)}=0$ is more difficult to satisfy than the convexity condition $\Omega^{(2)}>0$, which can be satisfied for any integrable cross section.

Cylinders are special because $\Omega^{(1)}=0$ by symmetry, so stability is determined solely by convexity, where surface and shell effects compete on an equal footing (cf. Fig. 1). In contrast, non-axisymmetric shapes can only occur for small $k_{F} \rho$. More precisely, the maximum deviation from axisymmetry is a decreasing function of $k_{F} \rho$ (see Figs. 2 and 3). Both $\delta \Omega^{(1)}$ and $\Omega_{s}^{(1)}$ vanish as $\lambda \rightarrow 0$, but whereas $\delta \Omega^{(1)}$ is an oscillatory function of $\lambda$ [cf. Eq. (5)], $\Omega_{s}^{(1)}$ grows smoothly with increasing $\lambda$ :

$$
\Omega_{s}^{(1)} / L \approx \sigma_{s} \lambda_{m}\left(m^{2}-1\right) 2 \pi \rho .
$$

The stationarity condition $\Omega^{(1)}=0$ can therefore only be satisfied for

$$
\left|\lambda_{m}\right|<\frac{\text { const. }}{m^{2}-1}\left(\frac{E_{F} k_{F}^{2}}{\sigma_{s}}\right)\left(k_{F} \rho\right)^{-\nu} .
$$

Indeed, the decrease in the maximum deformations as a function of $k_{F} \rho$ in Figs. 2, 3, and 4 is consistent with Eq. (32) with $3 / 2 \geq \nu>1$.

Thus cylinders are the only generically stable structures, and account for the principal peaks in conductance histograms of monovalent metals, while structures with broken symmetry are favored only at the nodes of the cylindrical supershell structure.

\section{Acknowledgments}

We thank F. Kassubek, H. Tureci, and A. Douglas Stone for useful discussions. D.F.U. and C.A.S. acknowledge the Aspen Center of Physics, where the final stage of this project was carried out. This work was supported by the DFG and the EU Training Network DIENOW (D.F.U. and H.G.) and by NSF Grant Nos. 0312028 (J.B. and C.A.S.) and 0351964 (J.B.).

\section{APPENDIX: ENERGY SPECTRUM OF A 2D-BILLIARD}

The essential input for the stability analysis of a nanowire (Sec. III) is the energy spectrum of a twodimensional billiard for which the boundary (in polar coordinates $r, \varphi)$ is given in terms of the radius function $R(\varphi ; \rho, \boldsymbol{\Lambda}) \equiv R_{\varphi}$ of the wire, by the condition $r=R_{\varphi}$. This provides the transverse eigenenergies $E_{n}(\rho, \boldsymbol{\Lambda})$ entering the expressions (18) and (19). It is helpful to introduce a change of coordinates, $\tilde{r}=r / R_{\varphi}$, which simplifies the boundary to a circle of unit radius. The Hamiltonian in the new coordinates $(\tilde{r}, \varphi)$ can be written as $H=H^{(0)}+\delta H$, where $H^{(0)}=-R_{\varphi}^{-2} \triangle_{\tilde{r}, \varphi}$ is the Hamiltonian for an axisymmetric problem and $\triangle_{\tilde{r}, \varphi}$ is the Laplace operator. For simplicity of notation we use units in which $\hbar^{2} / 2 m_{e}=1$. On the other hand, $\delta H$ contains the terms due to deviations from axial symmetry and is found to read

$$
\delta H=-\frac{R_{\varphi}^{\prime 2}}{R_{\varphi}^{4}} \frac{\partial^{2}}{\partial \tilde{r}^{2}}+2 \frac{R_{\varphi}^{\prime}}{R_{\varphi}^{3}} \frac{1}{\tilde{r}} \frac{\partial}{\partial \tilde{r}} \frac{\partial}{\partial \varphi}-\frac{2 R_{\varphi}^{\prime 2}-R_{\varphi}^{\prime \prime} R_{\varphi}}{R_{\varphi}^{4}} \frac{1}{\tilde{r}} \frac{\partial}{\partial \tilde{r}}
$$

where a prime indicates differentiation with respect to $\varphi$. The energy spectrum can be obtained by numerical diagonalization of $H$ in an appropriate basis, that we choose to be

$$
\Psi_{\mu \nu}(\tilde{r}, \varphi)=\frac{1}{\sqrt{\pi} \mathcal{J}_{\mu+1}\left(\gamma_{\mu \nu}\right)} e^{i \mu \varphi} \mathcal{J}_{\mu}\left(\gamma_{\mu \nu} \tilde{r}\right),
$$

where the $\gamma_{\mu \nu}$ are the roots of the Bessel functions $\mathcal{J}_{\mu}$. We simplify the notation by using roman indices $j$ as multi-indices representing a set of quantum numbers $\left(\mu_{j}, \nu_{j}\right)$. Note that the states (A.2), though normalized, are not orthogonal, since the scalar product in the new coordinates,

$$
\langle j \mid k\rangle:=\int_{0}^{2 \pi} R_{\varphi}^{2} d \varphi \int_{0}^{1} \tilde{r} d \tilde{r} \Psi_{j}^{*}(\tilde{r}, \varphi) \Psi_{k}(\tilde{r}, \varphi),
$$

involves a factor $R_{\varphi}^{2}$. However, the $\left\{\Psi_{j}\right\}$ still form a basis and the energy spectrum can be obtained by solving a generalized eigenvalue problem. Defining the matrices $\mathcal{H}_{j k}=\langle j|H| k\rangle$ and $\mathcal{B}_{j k}=\langle j \mid k\rangle$ we have to solve $\operatorname{det}[\mathcal{H}-E \mathcal{B}]=0$. Taking matrix elements of $\delta H$ we obtain after some algebra,

$$
\begin{aligned}
\langle j|\delta H| k\rangle= & \left\langle j\left|\left(\gamma_{j}^{2} \delta_{\gamma_{j}, \gamma_{k}}+\frac{\mu_{j}^{2} \gamma_{k}^{2}-\mu_{k}^{2} \gamma_{j}^{2}}{\gamma_{j}^{2}-\gamma_{k}^{2}} \frac{1}{\tilde{r}^{2}}\right) \frac{R_{\varphi}^{\prime 2}}{R_{\varphi}^{4}}\right| k\right\rangle \\
& +\left(\mu_{j}+\mu_{k}\right)\left\langle j\left|\frac{i R_{\varphi}^{\prime}}{R_{\varphi}^{3}} \frac{1}{\tilde{r}} \frac{\partial}{\partial \tilde{r}}\right| k\right\rangle
\end{aligned}
$$

where the terms could be simplified by making use of Bessel's equation.

We split the wave functions (A.2) into angular and radial parts, $\phi_{j}(\varphi)$ and $\chi_{j}(\tilde{r})$, respectively, and introduce 
the following matrices of $\tilde{r}$ - and $\varphi$-integrals

$$
\begin{array}{ll}
\mathcal{R}_{j k}^{[a]}=\int_{0}^{1} d \tilde{r} \tilde{r} \chi_{j}^{*} \chi_{k}, & \mathcal{I}_{j k}^{[a]}=\int_{0}^{2 \pi} d \varphi \phi_{j}^{*}\left(\frac{R_{\varphi}{ }^{\prime}}{R_{\varphi}}\right)^{2} \phi_{k}, \\
\mathcal{R}_{j k}^{[b]}=\int_{0}^{1} d \tilde{r} \frac{1}{\tilde{r}} \chi_{j}^{*} \chi_{k}, & \mathcal{I}_{j k}^{[b]}=\int_{0}^{2 \pi} d \varphi \phi_{j}^{*}\left(\frac{i R_{\varphi}{ }^{\prime}}{R_{\varphi}}\right) \phi_{k}, \\
\mathcal{R}_{j k}^{[c]}=\int_{0}^{1} d \tilde{r} \chi_{j}^{*} \frac{\partial \chi_{k}}{\partial \tilde{r}}, & \mathcal{I}_{j k}^{[c]}=\int_{0}^{2 \pi} d \varphi\left(\frac{R_{\varphi}}{\rho}\right)^{2} \phi_{j}^{*} \phi_{k},
\end{array}
$$

Note that the $\mathcal{R}^{[\cdot]}$ do not depend on the deformation parameters $(\rho, \boldsymbol{\Lambda})$ and that the $\mathcal{I}^{[.]}$only depend on $\boldsymbol{\Lambda}$. Decomposing the matrices $\mathcal{H}$ and $\mathcal{B}$ in terms of these newly defined matrices we find

$$
\begin{aligned}
& \mathcal{H}_{j k}= \\
& \begin{cases}\gamma_{j}^{2} \delta_{j k}+\left(\gamma_{j}^{2}-\mu_{j}^{2} \mathcal{R}_{j k}^{[b]}\right) \mathcal{I}_{j k}^{[a]} ; & \text { if } \gamma_{j}=\gamma_{k}, \\
\frac{\mu_{j}^{2} \gamma_{k}^{2}-\mu_{k}^{2} \gamma_{j}^{2}}{\gamma_{j}^{2}-\gamma_{k}^{2}} \mathcal{R}_{j k}^{[b]} \mathcal{I}_{j k}^{[a]}+\left(\mu_{j}+\mu_{k}\right) \mathcal{R}_{j k}^{[c]} \mathcal{I}_{j k}^{[b]} & \text { if } \gamma_{j} \neq \gamma_{k},\end{cases} \\
& \mathcal{B}_{j k}= \begin{cases}\rho^{2} & \text { if } j=k, \\
\rho^{2} \mathcal{R}_{j k}^{[a]} \mathcal{I}_{j k}^{[c]} & \text { otherwise. }\end{cases}
\end{aligned}
$$

These results can now be implemented to solve the generalized eigenvalue problem numerically. Obviously, the larger the deviations from axial symmetry, the more basis functions have to be used in order to achieve good convergence.

Expansion for small deformations of a cylinder: Moreover, Eqs. (A.6) and (A.7) also allow to study analytically the effect of a small deformation of a cylindrical wire on the transverse eigenenergies and the lifting of the degeneracies through the breaking of the rotational symmetry. Hereby we can give a proof of the relations (21-23) which ensure that the stability matrix of a cylindrical wire is always diagonal.

Let the initial shape be deformed according to the radius function (20). The deformation parameters are considered to be small so that we can expand the integrals $\mathcal{I}^{[.]}$up to second order in the $\lambda_{m}$. We find that they take non-zero values only if the quantum numbers $\mu_{j}$ and $\mu_{k}$ satisfy one of the following relations,

$$
\begin{aligned}
\mu_{k}-\mu_{j} & = \pm n \cdot m, \\
\left|\mu_{k}-\mu_{j}\right| & =|m \pm \bar{m}|,
\end{aligned}
$$

where $n$ is an integer and $m$ and $\bar{m}$ are elements from the set $\mathbb{M}$ of $m$-values appearing in the sum of Eq. (20). The results are summarized in Tab. III.

We can now straightforwardly derive the energy levels $E_{j}$ within perturbation theory of the generalized eigenvalue problem. Therefore we expand all the quantities entering the problem up to second order in the perturbation and solve order by order. For non-degenerate states

\begin{tabular}{|l|c|c|c|}
\hline & $\mathcal{I}_{j k}^{[a]}$ & $\mathcal{I}_{j k}^{[b]}$ & $\mathcal{I}_{j k}^{[c]}$ \\
\hline$\mu_{k}-\mu_{j}=0$ & $\sum_{m} m^{2} \lambda_{m}^{2} / 2$ & 0 & 1 \\
$\mu_{k}-\mu_{j}= \pm m$ & $\mathcal{O}\left(\lambda_{m}^{3}\right)$ & $\pm m \lambda_{m} / 2$ & $\lambda_{m}$ \\
$\mu_{k}-\mu_{j}= \pm 2 m$ & $-m^{2} \lambda_{m}^{2} / 4$ & $\mp m \lambda_{m}^{2} / 4$ & $\lambda_{m}^{2} / 4$ \\
$\left|\mu_{k}-\mu_{j}\right|=|m \pm \bar{m}|$ & $\mp \frac{m m^{\prime}}{2} \lambda_{m} \lambda_{\bar{m}}$ & $\frac{1}{2}\left(\mu_{j}-\mu_{k}\right) \lambda_{m} \lambda_{\bar{m}}$ & $\frac{1}{2} \lambda_{m} \lambda_{\bar{m}}$ \\
otherwise & $\mathcal{O}\left(\lambda_{m}^{3}\right)$ & $\mathcal{O}\left(\lambda_{m}^{3}\right)$ & 0 \\
\hline
\end{tabular}

TABLE III: Expansion of the integrals $\mathcal{I}^{[a]}, \mathcal{I}^{[b]}$, and $\mathcal{I}^{[c]}$ for small perturbations. Non-zero values are only found if one of the conditions given in the first column is satisfied.

$(\mu=0)$ it is found that

$$
\begin{aligned}
& \rho^{2} E_{j}^{(1)}=\mathcal{H}_{j j}^{(1)}-E_{j}^{(0)} \mathcal{B}_{j j}^{(1)} \\
& \rho^{2} E_{j}^{(2)}= \\
& \mathcal{H}_{j j}^{(2)}-E_{j}^{(1)} \mathcal{B}_{j j}^{(1)}-E_{j}^{(0)} \mathcal{B}_{j j}^{(2)}+\sum_{k \neq j} \frac{\mid \mathrm{A} .10)}{\mathcal{H}_{j k}^{(1)}-\left.E_{j}^{(0)} \mathcal{B}_{j k}^{(1)}\right|^{2}}(\mathrm{~A} .11) \\
& E_{k}^{(0)}-E_{k}^{(0)}
\end{aligned}
$$

where the matrix $\mathcal{B}$ in the equations above reflects the use of a non-orthogonal basis. From Eqs. (A.6) and (A.7) and the first row of Table III we deduce $\mathcal{H}_{j j}^{(1)}=\mathcal{B}_{j j}^{(1)}=$ $\mathcal{B}_{j j}^{(2)}=0$, so that $E_{j}^{(1)}=0$ and

$$
\begin{aligned}
& \rho^{2} E_{j}^{(2)}=\mathcal{H}_{j j}^{(2)}+\sum_{\substack{k \neq j \\
\left|\mu_{k}-\mu_{j}\right|=m}} \frac{\left|\mathcal{H}_{j k}^{(1)}-E_{j}^{(0)} \mathcal{B}_{j k}^{(1)}\right|^{2}}{E_{j}^{(0)}-E_{k}^{(0)}} \\
& =\sum_{m} \lambda_{m}^{2}\left(\frac{\gamma_{j}^{2}-\mu_{j}^{2} \mathcal{R}_{j j}^{[b]}}{2 / m^{2}}+\sum_{\substack{k \neq j \\
\left|\mu_{k}-\mu_{j}\right|=m}} \frac{\left|\frac{\mu_{j}^{2}-\mu_{k}^{2}}{2} \mathcal{R}_{j k}^{[c]}+\gamma_{j}^{2} \mathcal{R}_{j k}^{[a]}\right|^{2}}{\gamma_{j}^{2}-\gamma_{k}^{2}}\right) .
\end{aligned}
$$

Since $E_{j}^{(2)}$ does not contain mixed terms $\propto \lambda_{m} \lambda_{\bar{m}}$ (with $m \neq \bar{m}$ ) we have hereby derived the relation $(21)$.

The case $\mu>0$ is slightly more complicated as it requires degenerate perturbation theory: In first order we have to solve

$$
\left|\left(\begin{array}{ll}
\mathcal{H}_{i i}^{(1)} & \mathcal{H}_{i j}^{(1)} \\
\mathcal{H}_{j i}^{(1)} & \mathcal{H}_{j j}^{(1)}
\end{array}\right)-E^{(0)}\left(\begin{array}{ll}
\mathcal{B}_{i i}^{(1)} & \mathcal{B}_{i j}^{(1)} \\
\mathcal{B}_{j i}^{(1)} & \mathcal{B}_{j j}^{(1)}
\end{array}\right)-E^{(1)} \rho^{2} \mathbb{1}\right|=0
$$

where $\mathbb{1}$ is the unit matrix, and $i=(\mu, \nu)$, and $j=$ $(-\mu, \nu)$. Using the expansions given in Tab. III, we find

$$
E_{i}^{(1)}= \begin{cases} \pm\left(\gamma_{i} / \rho\right)^{2} & \text { if } 2 \mu=m \\ 0 & \text { otherwise }\end{cases}
$$

The energy change in second order degenerate perturbation theory is found to read

$$
E_{i}^{(2)}=\mathcal{H}_{i i}^{(2)}+\mathcal{C}_{i i} \mp\left(\mathcal{H}_{i j}^{(2)}-E_{i}^{(1)} \mathcal{B}_{i j}^{(1)}-E_{i}^{(0)} \mathcal{B}_{i j}^{(2)}+\mathcal{C}_{i j}\right)(
$$


where we have used $\mathcal{B}_{i i}^{(1)}=\mathcal{B}_{i i}^{(2)}=0$, and the matrix

$$
\mathcal{C}_{i j}=\sum_{k \neq i, j} \frac{\left(\mathcal{H}_{j k}^{(1)}-E_{i}^{(0)} \mathcal{B}_{j k}^{(1)}\right)\left(\mathcal{H}_{i k}^{(1)}-E_{i}^{(0)} \mathcal{B}_{i k}^{(1)}\right)}{E_{i}^{(0)}-E_{k}^{(0)}} .(\mathrm{A} .16)
$$

Note that the quantity $\mathcal{H}_{i i}^{(2)}+\mathcal{C}_{i i}$ appearing in Eq. (A.15) is equal to the full result (A.12) for the non-degenerate case. Therefore we conclude that relations (22) and (23) hold.
1 N. Agraït, A. Levy Yeyati, and J. M. van Ruitenbeek, Phys. Rep. 377, 81 (2003), and refs. therein.

2 G. Rubio, N. Agraït, and S. Vieira, Phys. Rev. Lett. 76, 2302 (1996).

${ }^{3}$ C. Untiedt, G. Rubio, S. Vieira, and N. Agraït, Phys. Rev. B 56, 2154 (1997).

${ }^{4}$ Y. Kondo and K. Takayanagi, Phys. Rev. Lett. 79, 3455 (1997).

5 V. Rodrigues, T. Fuhrer, and D. Ugarte, Phys. Rev. Lett. 85, 4124 (2000).

${ }^{6}$ Y. Kondo and K. Takayanagi, Science 289, 606 (2000).

7 A. I. Yanson, I. K. Yanson, and J. M. van Ruitenbeek, Nature 400, 144 (1999).

8 A. I. Yanson, I. K. Yanson, and J. M. van Ruitenbeek, Phys. Rev. Lett. 84, 5832 (2000).

9 A. I. Yanson, I. K. Yanson, and J. M. van Ruitenbeek, Fizika Nizkikh Temperatur 27, 1092 (2001).

10 M. Díaz, J. L. Costa-Krämer, E. Medina, A. Hasmy, and P. A. Serena, Nanotech. 14, 113 (2003).

11 A. I. Mares, A. F. Otte, L. G. Soukiassian, R. H. M. Smit, and J. M. van Ruitenbeek, Phys. Rev. B 70, 073401 (2004).

12 A. I. Mares and J. M. van Ruitenbeek, Phys. Rev. B 72, $205402(2005)$.

13 C. H. Zhang, F. Kassubek, and C. A. Stafford, Phys. Rev. B 68, 165414 (2003).

14 S. Chandrasekhar, Hydrodynamic and Hydromagnetic Stability (Dover, New York, 1981), pp. 515-74.

15 W. A. de Heer, Rev. Mod. Phys. 65, 611 (1993).

16 C. A. Stafford, D. Baeriswyl, and J. Bürki, Phys. Rev. Lett. 79, 2863 (1997).

17 J. Bürki and C. A. Stafford, Applied Physics A 81, 1519 (2005).

18 F. Kassubek, C. A. Stafford, H. Grabert, and R. E. Goldstein, Nonlinearity 14, 167 (2001).

19 D. F. Urban and H. Grabert, Phys. Rev. Lett. 91, 256803 (2003).

20 J. Bürki, R. E. Goldstein, and C. A. Stafford, Phys. Rev. Lett. 91, 254501 (2003).

21 J. Bürki, C. A. Stafford, and D. L. Stein, Phys. Rev. Lett. 95, 090601 (2005).

22 A. Bulgac and C. Lewenkopf, Phys. Rev. Lett. 71, 4130 (1993).

23 M. Schmidt, C. Ellert, W. Kronmüller, and H. Haberland, Phys. Rev. B 59, 10970 (1999).

24 D. F. Urban, J. Bürki, C. H. Zhang, C. A. Stafford, and H. Grabert, Phys. Rev. Lett. 93, 186403 (2004).
25 D. F. Urban, J. Bürki, A. I. Yanson, I. K. Yanson, C. A. Stafford, J. M. van Ruitenbeek, and H. Grabert, Solid State Comm. 131, 609 (2004).

26 M. Brack and R. K. Bhaduri, Semiclassical Physics, vol. 96 of Frontiers in Physics (Addison-Wesley, Reading, MA, 1997).

27 M. Brack, Rev. Mod. Phys. 65, 677 (1993).

${ }^{28}$ F. Kassubek, C. A. Stafford, and H. Grabert, Phys. Rev. B 59, 7560 (1999).

29 C.-H. Zhang, J. Bürki, and C. A. Stafford, Phys. Rev. B 71, 235404 (2005).

30 A. García-Martin, J. A. Torres, and J. J. Sáenz, Phys. Rev. B 54, 13448 (1996).

31 T. P. Martin, Phys. Rep. 273, 199 (1996).

32 R. Dashen, S.-K. Ma, and H. J. Bernstein, Phys. Rev. 187, 345 (1969).

33 V. M. Strutinsky, Nucl. Phys. A 122, 1 (1968).

34 C. A. Stafford, F. Kassubek, J. Bürki, and H. Grabert, Phys. Rev. Lett. 83, 4836 (1999).

35 M. C. Gutzwiller, Chaos in Classical and Quantum Mechanics (Springer, New York, 1990).

36 M. C. Gutzwiller, J. Math. Phys. 12, 343 (1971).

37 C. A. Stafford, F. Kassubek, and H. Grabert, Adv. Solid State Phys. 41, 497 (2001).

38 D. F. Urban, C. A. Stafford, and H. Grabert, Scaling theory of $C D W$ in multi-channel metal nanowires, preprint (2006).

39 J. P. Perdew, Y. Wang, and E. Engel, Phys. Rev. Lett. 66, 508 (1991).

40 K. Huang and G. Wyllie, Proc. Phys. Soc. A 62, 180 (1949).

41 H. B. Huntington, Phys. Rev. 81, 1035 (1951).

42 G. D. Mahan, Phys. Rev. B 12, 5585 (1975).

43 Note that surface tension and curvature energy experimentally depend on temperature. This can be accounted for by making $\eta$ and $\xi$ temperature dependent, but does not significantly alter the results.

44 N. W. Ashcroft and N. D. Mermin, Solid State Physics (Saunders College Publishing, 1976).

45 A. G. Magner, S. N. Fedotkin, F. A. Ivanyuk, P. Meier, M. Brack, S. M. Reimann, and H. Koizumi, Ann. Phys. (Leipzig) 6, 555 (1997).

${ }^{46}$ K. Richter, D. Ullmo, and R. A. Jalabert, Phys. Rep. 276, 1 (1996). 